
\title{
3 Research S Suare \\ The intervention of S1PR1/STAT3 signaling pathway by inhibiting the expression of STAT3 attenuates the valvular damage due to rheumatic heart disease
}

\section{Shenglin Xian}

The First Affiliated Hospital of Guangxi Medical University

\section{Ang Chen}

The First Affiliated Hospital of Guangxi Medical University

\section{Yunjiao Wu}

The First Affiliated Hospital of Guangxi Medical University

\section{Hong Wen}

The First Affiliated Hospital of Guangxi Medical University

\section{Chuanghong Lu}

The First Affiliated Hospital of Guangxi Medical University

Jianlin Wen

The First Affiliated Hospital of Guangxi Medical University

\section{Feng Huang}

The First Affiliated Hospital of Guangxi Medical University

\section{Zhiyu Zeng ( $\nabla$ drzyzeng@126.com )}

The First Affiliated Hospital of Guangxi Medical University https://orcid.org/0000-0002-7383-2700

\section{Research article}

Keywords: rheumatic heart disease, valvular damage, T helper 17 cell, signal transducer and activator of transcription 3

Posted Date: June 2nd, 2020

DOl: https://doi.org/10.21203/rs.3.rs-31680/v1

License: (c) (1) This work is licensed under a Creative Commons Attribution 4.0 International License. Read Full License 


\section{Abstract \\ Background}

Rheumatic heart disease (RHD) is a social health problem that affects many patients every year, but its pathogenesis is still unclear. Recent studies have found that the sphingosine 1-phosphate receptor 1 (S1PR1)/signal transducer and activator of transcription 3 (STAT3) signaling pathway is activated during the development of valvular damage caused by RHD. STAT3 plays an important role in the differentiation of CD4 + T cells into T helper 17 (Th17) cells, and Th17 cell-related cytokines are involved in the development of RHD. In this work, we investigated whether altering the S1PR1/STAT3 signaling pathway by inhibiting the expression of STAT3 attenuates valvular damage due to RHD.

\section{Methods}

Inactivated Group A streptococci (GAS) and complete Freund's adjuvant (CFA) were used to establish the RHD rat model. STAT3-small interfering RNA (STAT3-siRNA) was used to directly inhibit the expression of STAT3 in the heart. Histological examination was used to evaluate the degree of inflammation and fibrosis in valve tissues. Enzyme-linked immunosorbent assay (ELISA) and immunohistochemistry were used to detect the levels of interleukin (IL)- 6 and IL-17 in serum and valve tissue. Reverse transcriptionquantitative PCR (RT-qPCR) was used to detect the mRNA expression of S1PR1 and STAT3. Western blotting (WB) and immunohistochemistry were used to detect the protein expression of S1PR1, STAT3, phosphorylated (p-) STAT3, retinoic acid-related orphan receptor gamma T (RORYt).

\section{Results}

The expression of S1PR1 in valve tissues of RHD model rats was decreased. The degree of valvular inflammation and fibrosis of RHD model rats was increased. The levels of IL- 6 and IL-17 in valves and serum of RHD model rats were increased as well as an increase of p-STAT3 in valve tissues. Inhibition of STAT3 by STAT3-siRNA decreased STAT3 expression and reduced the total amount of p-STAT3, resulting in decreased expression of IL-6, IL-17 and RORyt in valves and serum and reduced valvular inflammatory response and fibrosis.

\section{Conclusions}

These results suggest that inhibiting the expression of STAT3 in the heart may reduce the valvular damage caused by rheumatic heart disease.

\section{Background}


RHD is a preventable heart disease that is caused by Streptococcus pyogenes or group A streptococcal (GAS) infection [1]. RHD continues to be a leading cause of deaths and disability in young patients and remains a serious public health problem worldwide [2]. At present, the number of people with RHD disability in the world exceeds one quarter of the number of people with cancer disability, and the number of deaths caused by RHD each year is as high as 250,000 [3]. However, the research progress on RHD is still exploring the pathogenesis, which is still unclear. There are no precise intervention targets for the development of RHD. Most RHD research is about the relationship between pathogenesis and signaling pathways [4-6]. There is a lack of research on intervention targets that can attenuate valvular damage due to RHD.

STAT3 is a cellular signal transcription factor involved in the regulation of many cellular activities [7]. STAT3 may regulate the differentiation of $\mathrm{CD} 4^{+} \mathrm{T}$ cells into Th17 cells [8]. Several studies have shown that Th17 cells and related cytokines mediate inflammatory responses and autoimmune responses [911]. Studies by Bas et al. showed that both the ratio of Th17/Treg cells and the level of IL-17A in patients with RHD were significantly increased than those in the control group [12]. Similar results were found in RHD animal models using Lewis rats [13].

S1PR1 is a G protein-coupled receptor belonging to sphingosine 1-phosphate receptor (S1PR) family. S1PR1 mediates lymphocyte migration and is associated with multiple immune diseases [14], and a previous study found that downregulation of S1PR1 expression increases phosphorylation levels of STAT3 [15]. A recent study discovered that the S1PR1/STAT3 signaling pathway is activated during the development of vavular damage due to RHD from the study on RHD model rats [16]. Therefore, we hypothesize that the STAT3 pathway is activated in the development of RHD, which induces CD $4^{+} \mathrm{T}$ cells to differentiate into Th17 cells, and then Th17 cell-related inflammatory factors participate in the development of RHD. However, it is unclear whether this pathway influences or prevents RHD after intervention. What is more important is how to intervene in this pathway to influence or prevent the occurrence of RHD. Therefore, in this study, we interfered with this pathway by inhibiting the expression of STAT3.

In this study, we interfered with S1PR1/STAT3 signaling pathway by inhibiting the expression of STAT3 to determine whether it could attenuate the valvular damage caused by RHD.

\section{Methods}

In this study, we aimed to find out whether inhibiting the expression of STAT3 in the heart can attenuate the valvular damage caused by RHD. In order to achieve this goal, RHD rat model was established, the expression of STAT3 in the heart was inhibited by STAT3-siRNA, Th17 cell-related inflammatory factors were detected. Then, the degree of inflammation and fibrosis in valve tissues was evaluated to finally verify our conjecture.

\subsection{Antigen}


First, brain heart infusion fluid medium (Guangdong Huankai Microbial Sci. \& Tech. Co., Ltd.) was used to culture the group A streptococci (GAS, ATCC19615; American Type Culture Collection), the temperature during cultivation process was kept constant at $37^{\circ} \mathrm{C}$. After $24 \mathrm{~h}$, the GAS were washed with normal saline (NS), and then transferred into $10 \%$ neutral formalin for $12 \mathrm{~h}$ for inactivation. Second, NS was used to wash and resuspend the inactivated GAS, and the concentration was simultaneously adjusted to $4.0 \times$ $10^{11} \mathrm{CFU} / \mathrm{ml}$. Finally, after emulsifying the suspensions by sonication (Sonics \& Materials, Inc.), we obtained the antigen.

\subsection{In vivo gene therapy}

In order to directly inhibit the expression of STAT3 in the heart of Lewis rats, a rat STAT3 small interfering RNA (siRNA) sequence (5'-GGCTGATCATTTATATAAA-3') with a c-TNT promoter (STAT3-siRNA; Hanbio Biotechnology Co., Ltd.) carried by recombinant adeno-associated viral (AAV, serotype 9) vectors was used. In addition, to determine whether the AAV vector had an effect on the rats, we used an AAV-control as a negative control.

\subsection{Animals and groups}

We purchased 24 Lewis rats (150-180 g) from Beijing Vital River Animal Technology Co., Ltd. All the rats were female and at 8 weeks of age, and weighing 150-180 g. Our pathogen-free animal laboratory at the Animal Experiment Center of Guangxi Medical University provides a good environment for captive rats: the temperature is constantly set to $23^{\circ} \mathrm{C}$ and the fluctuation does not exceed $2^{\circ} \mathrm{C}$; the cycle of day and night is 12 hours; the movement of rats in the cage is completely unrestricted; and we provide convenient drinking conditions and standard rat feed. We allowed all the rats to adapt to the environment for 5 days before the start of the experiment. All animal experimental procedures were conducted according to the ethical guidelines for the care and use of laboratory animals and were approved by the Medical Ethics Committee of the First Affiliated Hospital of Guangxi Medical University (grant no. 2019-KY-E-034). All rats were divided randomly into four groups: control group, STAT3-siRNA group, AAV-control group and RHD group. Each group had 6 rats. The RHD group was the established RHD model. Footpad injection of CFA (Sigma-Aldrich, Merck KGaA) is essential in establishing the RHD rat model. And all rats were maintained on soft bedding and not in wire-bottomed cages. We used 9 weeks to establish the RHD rat model. In the beginning, one hind footpad of the rat was injected with a $100 \mu$ l solution of inactivated GAS $\left(4.0 \times 10^{11} \mathrm{CFU} / \mathrm{ml}\right)$ and CFA mixed at a ratio of 1:1 (v/v). Then, after one week, we performed a subcutaneous injection of $500 \mu$ of inactivated GAS $\left(4.0 \times 10^{11} \mathrm{CFU} / \mathrm{ml}\right)$ and CFA mixed at a ratio of 1:1 $(\mathrm{v} / \mathrm{v})$ into the abdomen once a week at the same interval for 4 weeks. For the last 4 weeks, we performed subcutaneous abdominal injection once a week at the same interval with an adjustment of the injection solution to $500 \mu \mathrm{l}$ of inactivated GAS $\left(4.0 \times 10^{11} \mathrm{CFU} / \mathrm{ml}\right)$. Rats in the STAT3-siRNA group were injected with $2.5 \times 10^{11}$ viral genome particles at one time through the tail vein (AAV-STAT3-siRNA, diluted with $200 \mu \mathrm{l}$ normal saline) at the beginning of the experiment. Three weeks later, the rats received exactly the same treatment as the RHD group. The rats in the AAV-control group received an injection of $2.5 \times 10^{11}$ viral genome particles at one time through the tail vein (AAV-control, diluted with $200 \mu \mathrm{l}$ normal saline) at 
the beginning of the experiment. Three weeks later, they were injected following the same protocol as that of the RHD group. The rats in the control group were injected using the same protocol as that of the RHD group from the beginning, but the injection solution was the same volume of NS.

\subsection{Sacrifice}

After the treatments, we collected a total of $1 \mathrm{ml}$ blood via tail veins of rats from each group without anaesthesia, then an intraperitoneal injection of sodium pentobarbital $(150 \mathrm{mg} / \mathrm{kg})$ was used to sacrifice the rat. Animal death was determined when there was more than 5 min without breathing or heartbeat. Humane endpoint was defined as animals losing $>15 \%$ of their body weight with a decreased ability to consume food and water

\subsection{Sample preparation}

We collected samples from the valves of every rat. All samples were frozen in liquid nitrogen rapidly and then stored at $-80^{\circ} \mathrm{C}$ for follow-up experiments. Since no animals died during the modeling process, there were 6 samples in each group, for a total of 24 samples. The next five experimental methods (histochemistry, immunohistochemistry, RT-qPCR, WB, ELISA) were based on the previously mentioned experimental grouping and tested 6 samples in each group.

\subsection{Histochemistry}

The valve tissues were fixed for $24 \mathrm{~h}$ at $4{ }^{\circ} \mathrm{C}$ in $4 \%$ paraformaldehyde before decalcifying and embedding in paraffin blocks. All blocks were serially sectioned at $5 \mu \mathrm{m}$ for haematoxylin and eosin (H\&E) staining and Sirius red staining. H\&E staining was performed at room temperature, the sections were first stained with haematoxylin for 4-10 min and then with eosin for 0.5-2 min. Then, in order to capture the images reflecting the results of H\&E staining, a BX43 light microscope (Olympus Corporation) was used. Sirius red staining was also performed at room temperature, the Sirius red solution was used to stain the sections for $1 \mathrm{~h}$. Then, in order to capture the images reflecting the results of Sirius red staining, a BX43 confocal microscope (magnification, $\times 100$; Olympus Corporation) was used.

\subsection{Immunohistochemistry}

Immunohistochemistry was performed following a previous report [17] to analyse the valve tissues, which were stained for IL-6 (1:65; cat. no. ab9324; Abcam), IL-17 (1:90; cat. no. ab214588; Abcam), S1PR1 (1:80; cat. no. ab77076; Abcam), STAT3 (1:75; cat. no. ab69153; Abcam), p-STAT3 (1:70; cat. no. ab76315; Abcam). And RORyt (1:75; cat. no. 13205-1-AP; Proteintech) which is the key transcription factor that is driving the differentiation of IL-17 producing Th17 cells [18] was also detected. Briefly, the valve tissues were embedded in formalin-fixed paraffin first. Then all blocks were sectioned at $5 \mu \mathrm{m}$. After deparaffinization and rehydration, the $5 \%$ bovine serum albumin (BSA; Beijing Solarbio Science \& Technology Co., Ltd.) solution was used to block the sections at room temperature for $1 \mathrm{~h}$. After deactivation of endogenous peroxidase with hydrogen peroxide, the sections were incubated with the previously mentioned primary antibodies for $12 \mathrm{~h}$ at $4{ }^{\circ} \mathrm{C}$. Then, at room temperature, the anti-rabbit horseradish peroxidase (HRP)-conjugated (1:10; cat. no. PV-6001; OriGene Technologies, Inc.) or anti- 
mouse HRP-conjugated (1:10; cat. no. PV-6002; OriGene Technologies, Inc.) was used as secondary antibodies to incubate the sections for $30 \mathrm{~min}$. After enhancement of colour development by using diaminobenzidine (DAB), the immunostained tissues were examined under a BX43 light microscope (Olympus Corporation), and positive expression was reflected by brownish yellow staining, which was detected by microscopy. Quantitative assessment was performed using the methods provided by Friedrichs et al. in a previous report [19]. The positive cell percentage and immunohistochemical score (IHS) were also used in our experiment to describe the quantitative evaluation results.

\subsection{RT-qPCR}

First, total RNA was extracted from each sample. We used TRIzol® reagent (Invitrogen; Thermo Fisher Scientific, Inc.) according to the protocol supplied by the manufacturer to finish this step. Second, the concentration of RNA must be measured for quantitative reverse transcription. We used a NanoDrop ${ }^{T M}$ 2000 spectrophotometer (NanoDrop Technologies; Thermo Fisher Scientific, Inc.) to finish this step. Third, reverse transcription of RNA into cDNA. In this step we reverse transcribed $0.5 \mu \mathrm{g}$ of total RNA from each sample into cDNA. The kit we used was PrimeScript RT reagent kit (Takara Bio, Inc.). And the entire reverse transcription process is strictly in accordance with the instructions supplied by the manufacturer. Finally, RT-qPCR was performed. In this step, the kit we used was TB Green Premix Ex Taq $\otimes$ (Takara Bio, Inc.), the instrument we used was a StepOne system (Applied Biosystems; Thermo Fisher Scientific, Inc.), and the internal reference was set to $\beta$-actin, and the entire process was still strictly in accordance with the manufacturer's instructions. In Table 1 we list the sequences of the primer sequences in detail. The final result was expressed by the fold difference between the expression level of each mRNA and that of the internal reference using the $2^{\Delta \Delta C t}$ method [20]. All samples were measured 3 times. 
Table 1

Sequences of primers used in reverse transcription-quantitative PCR.

\begin{tabular}{|ll|}
\hline Gene & Primer sequence (5'-3') \\
\hline STAT3 & Forward: TTTGAGACAGAGGTGTACCACCAAG \\
\hline S1PR1 & Reverse: ACCACAGGATTGATGCCCAAG \\
\hline Col3a1 & Feverse: TTCTCCCTTCCCTCCCTCTC \\
FSP1 & Forward: ACTTCTGGTCCTCCTGGTCTGC \\
& Forward: TGGGGAGAAGGACAGACGAAGC \\
\hline B-actin & Feverse: TGGCAATGCAGGACAGGAAGAC \\
\hline & Reverse: GACTCATCGTACTCCTGCTTGCTG \\
\hline $\begin{array}{l}\text { In Table 1 we list the sequences of the primer sequences in detail. STAT3, signal transducer and } \\
\text { activator of transcription 3; S1PR1, sphingosine-1-phosphate receptor 1; Col3a1, collagen type III a1 } \\
\text { chain; FSP1, fibroblast-specific protein 1. }\end{array}$ \\
\hline
\end{tabular}

\subsection{Western blotting}

First, total protein was extracted from each sample. In this step, the kit we used was RIPA lysis buffer (Sangon Biotech Co., Ltd.), and the method used is according to the instruction supplied by the manufacturer. Second, the protein concentration was measured. In this step, the kit we used was a bicinchoninic acid (BCA) protein assay kit (Sangon Biotech Co., Ltd.). Third, the same amounts of protein $(30 \mu \mathrm{g})$ from each sample were separated. In this step, the material we used to separate the protein was $10 \%$ SDS-PAGE. The separation conditions were: $80 \mathrm{~V}$ for $30 \mathrm{~min}$ and $120 \mathrm{~V}$ for $60 \mathrm{~min}$, the equipment we used was a blotting system (Bio-Rad Laboratories, Inc.), and the entire process was still strictly in accordance with the manufacturer's instructions. Third, the separated proteins were electrotransferred to $0.22 \mu \mathrm{m}$ polyvinylidene fluoride (PVDF) membranes (EMD Millipore), the transfer conditions were: constant $80 \mathrm{~V}$ for $80 \mathrm{~min}$. Fourth, we blocked the membranes carrying proteins for $1 \mathrm{~h}$ at room temperature with 3\% BSA blocking solution (Sangon Biotech Co., Ltd.) and then incubated the membranes overnight at $4{ }^{\circ} \mathrm{C}$ with the following antibodies: anti-S1PR1 (1:1,000; cat. no. 55133-1-AP; ProteinTech Group, Inc.), anti-STAT3 (1:1,000; cat. no. ab68153; Abcam), anti-p-STAT3 (1:1,000; cat. no. 9145; Cell Signaling Technology, Inc.) and anti- $\beta$-tubulin (1:3,000; cat. no. 10068-1-AP; ProteinTech Group, Inc.). After that, at room temperature, we incubated the membranes in the dark for $1 \mathrm{~h}$ with HRPconjugated secondary antibody (10,000; cat. no. ab6721; Abcam). Finally, the protein bands were scanned by a chemiluminescence imaging system (Alpha FluorChem FC3; Alpha, Inc.) and we used 
ImageJ software (1.51j, National Institute of Health) to quantify the expression of the proteins, which were normalized to $\beta$-tubulin. All samples were measured 3 times.

\subsection{Enzyme-linked immunosorbent assay}

Enzyme-linked immunosorbent assay (ELISA) kits (cat. nos. E04640r and E07451r; Cusabio) were used to measure the levels of IL- 6 and IL-17 in the serum. The entire process was strictly in accordance with the manufacturer's instructions. All samples were measured 3 times.

\subsection{Statistics}

Results are expressed as the mean \pm standard deviation of at least three independent experiments. The software we used for statistical analysis was SPSS software 16.0 (SPSS, Inc.). The method we used to compare the differences between 2 groups is Student's t-test. The method we used when comparing the differences among 4 groups was one-way ANOVA. We used $p<0.05$ as the criterion for identifying significant differences.

\section{Results}

\subsection{STAT3-siRNA pretreatment decreased STAT3 expression and reduced the total amount of p-STAT3}

The result of RT-qPCR showed that compared with the control group, the expression of S1PR1 mRNA decreased in the three groups: AAV-control, RHD and STAT3-siRNA groups $(P<0.05 ;$ Fig. 1A). The results of western blotting and immunohistochemistry showed that the protein expression of S1PR1 was downregulated in the AAV-control, STAT3-siRNA and RHD groups compared with that of the control group $(P<0.05$; Fig. 2A, 2B and 3A-C). These results indicate that the expression of S1PR1 is down-regulated in the RHD rat model, which is consistent with previous reports $[16,17]$. Inhibition of STAT3 by STAT3-siRNA decreased STAT3 mRNA expression, this result can be reflected in the comparison between STAT3-siRNA group and the other three groups $(\mathrm{P}<0.05$; Fig. 1B). The protein expression of p-STAT3 was significantly higher in the RHD and AAV-control groups than in the control group $(P<0.05)$. And inhibition of STAT3 by STAT3-siRNA decreased the protein expression of STAT3, p-STAT3 in the valve tissues, this result can be reflected in the comparison between STAT3-siRNA group and the other three groups $(P<0.05$; Fig. 2A, 2C, 2D, 3A-C). And the ratio of $p-S T A T 3 /$ total ( $t$-) STAT3 was significantly higher in the STAT3-siRNA, AAVcontrol and RHD groups than in the control group $(P<0.05)$, while there was no significant difference among the STAT3-siRNA, AAV-control and RHD groups ( $P<0.05$; Fig. 2E and 3D), which indicates that STAT3-siRNA will not change the ratio of p-STAT3/t-STAT3, but will reduce the total amount of both STAT3 and p-STAT3. 


\subsection{The expression of Th17-related transcription factor and cytokines was reduced in STAT3-siRNA group than in RHD group}

The results of immunohistochemistry and ELISA showed that the expression of RORYt, IL-6 and IL-17 was significantly higher in the RHD and AAV-control groups than in the control group $(P<0.05)$. And inhibition of STAT3 by STAT3-siRNA decreased the expression of RORyt, IL-6 and IL-17 in the serum and valve tissues, this result can be reflected in the comparison between STAT3-siRNA group and the other three groups $(P<0.05$; Fig. 4).

\subsection{STAT3-siRNA pretreatment attenuates the valvular damage due to rheumatic heart disease}

H\&E staining showed that there was an inflammatory response in the heart valves of the rats in the AAVcontrol and RHD groups. In the STAT3-siRNA group, we observed that the inflammatory response is reduced compared to the RHD group (Fig. 5A). There was no such change in the heart valves of the rats in the control group. All changes were observed under a microscope. Sirius red staining has the ability to distinguish different types of collagen fibres. Previous studies have shown that type 1 collagen (COL1) fibres are the main type of collagen in nonfibrotic valves [21]. The ratio of type 3 collagen (COL3) and COL1 can be used to reflect the degree of fibrosis of the valve tissue. In the process of fibrosis of the valve tissue, the proportion of COL3 will become larger and larger, so the larger this ratio is, the more severe the fibrosis is. Therefore, we used the COL3/COL1 (COL3/1) ratio to quantify the degree of fibrosis of the valve tissue. In the pictures of Sirius red staining, type 1 collagen fibres are closely packed yellow and red fibres, with obvious birefringence, while type 3 collagen fibres are loosely arranged green fibres, with weak birefringence. The pictures showed that the fibrosis in RHD group is significantly more severe than that in control group. Accordingly, the COL3/1 ratio is also significantly higher in RHD group than in control group. The degree of fibrosis in the STAT3-siRNA group was lower than that in the RHD group. Accordingly, the COL3/1 ratio was significantly different between the STAT3-siRNA and RHD groups $(\mathrm{P}<$ 0.05; Fig. 5B and $5 \mathrm{C}$ ). In reflecting the fibrosis of the valve at the molecular level, we chose to detect two molecular markers: collagen type III a1 chain (Col3a1) and fibroblast-specific protein 1 (FSP1). And the results were consistent with the results of the histological examination $(P<0.05$; Fig. 5D and 5E). Both results reflected that after inhibiting the expression of STAT3, the inflammatory response and fibrosis of the valve were reduced compared with the RHD group.

\section{Discussion}

RHD has a long history, and many patients have been killed by this disease. RHD caused 319,400 deaths in 2015 [22], 314,600 deaths in 2016 [23] and 285,500 deaths in 2017 [24], but the pathogenesis of this disease is still unknown. In recent years, research progress has mostly focused on the signaling pathways that are related to pathogenesis. Through the efforts of researchers, some signaling pathways 
related to this disease have been discovered. Recently, researchers have discovered that the S1PR1/STAT3 signaling pathway is activated during vavular damage due to RHD from the study on RHD model rats [16]. However, it is unknown how intervening in the S1PR1/STAT3 pathway affects valvular damage caused by RHD. More importantly, which method can be used to intervene in the S1PR1/STAT3 pathway and effectively attenuate valvular damage due to RHD?

Previous research on the S1PR1/STAT3 signaling pathway found that S1PR1 gene deficiency led to increased STAT3 phosphorylation and activation of the IL-6-Jak-STAT3 molecular pathway in a mouse autoimmune encephalitis model with an S1PR1 gene mutation, thereby mediating Th17 cell differentiation [15]. STAT3 plays a key pathogenic role in many inflammatory conditions. Researchers have found that STAT3 mediates immune myocarditis due to IL-6 production-induced liver complement component C3 and Th17 cell differentiation [25], and the differentiation of Th17 cells plays an important role in the occurrence and development of myocarditis [26]. Furthermore, tissue signaling cytokines such as IL-22 and IL-17 have been proposed to have actions on the heart that involve STAT3 [27]. Previous studies have shown high expression of p-STAT3 in rheumatoid arthritis [28], and so we consider STAT3 to be the key part of this signaling pathway. Since STAT3 is overexpressed in this pathway, we wondered whether suppressing its expression can attenuate valvular damage due to rheumatic heart disease.

Therefore, we designed experiments to verify our hypotheses. The results of this study validated our speculation. The RT-qPCR, western blotting, and immunohistochemistry results in the control and RHD groups showed activation of the S1PR1/STAT3 signaling pathway in RHD. The RT-qPCR, western blotting, and immunohistochemistry results in the RHD and STAT3-siRNA groups suggest that AAV-STAT3-siRNA successfully inhibited the expression of STAT3. The histochemistry, immunohistochemistry and ELISA results in the RHD and STAT3-siRNA groups suggest that inhibition of STAT3 expression attenuates valvular damage due to RHD.

At present, the research progress on RHD is mainly focused on the discovery of signaling pathways that are activated during pathogenesis, such as research on the involvement of mitogen-activated protein kinases (MAPKs)/TGF- $\beta 1 /$ TRAF6 signaling pathway in RHD [29]. Studies that effectively affect the pathogenesis of RHD are still rare. Previous researchers have discovered that activation and high phosphorylation of STAT3 are involved in the differentiation of CD4 ${ }^{+} \mathrm{T}$ cells; more importantly, the direction of this differentiation is towards Th17 cell-associated effectors [30]. And previous studies have confirmed that the level of the Th17 cell-associated effector IL-17 in the serum of rheumatic mitral stenosis patients was significantly increased [31]. In RHD animal models using Lewis rats, the levels of IL-6 and IL-17 in mitral valve and serum samples were significantly higher than those in the control group [13]. Therefore, we envisaged whether it is possible to indirectly inhibit the phosphorylation of STAT3 by directly inhibiting the expression of STAT3, thereby interfering CD4 + T cells from differentiating into Th17 cells, reducing the levels of Th17 cell-associated cytokines, which could help to reduce RHD-induced valvular damage. 
The experimental results showed that after we specifically inhibited the expression of STAT3, the expression of the final key step of this pathway was blocked, and the total amount of p-STAT3 was significantly lower than that in the RHD group; therefore, the level of Th17 cell-associated effectors in the rats in the STAT3-siRNA group was significantly lower than that in the RHD group. The levels of inflammation and fibrosis in the STAT3-siRNA group were significantly reduced compared with that in the RHD group. STAT3 is also involved in many diseases, such as airway inflammation and rheumatoid arthritis [32-35]. The persistent activation and high expression of STAT3 in tumour cells promotes tumour growth and proliferation [7]. And sustained activation of STAT3 also mediates tumour-promoting inflammation [36]. Taking these findings into consideration, we believe that inhibiting the expression of STAT3 attenuates valvular damage due to RHD.

There are some limitations in our research. This was a rat study, and further research on humans is required. How will up-regulating the expression of STAT3 affect RHD is unknown. Cell experiments may provide cell-level evidence for our research, but we have not conducted it. Perhaps we can further understand the effect of the S1PR1/STAT3 signaling pathway on RHD by altering the expression of S1PR1. In addition, we did not detect the expression of FSP1 and Col3a1 at the protein level.

\section{Conclusion}

Therefore, the status of RHD is still severe, and although primary and secondary prevention strategies are clear, global implementation is not ideal [37]. Many researchers have made efforts, and its pathogenesis is still not understood. Studies on RHD have mostly confirmed that one of its signaling pathways is activated during its onset, while there have been few studies on how to affect the pathogenesis of RHD. By summarizing previous research results and the inflammatory mechanism of RHD pathogenesis, we hypothesize that altering the S1PR1/STAT3 signaling pathway may affect the process of valvular damage caused by RHD. Animal experiments showed that altering the S1PR1/STAT3 signaling pathway by inhibiting the expression of STAT3 attenuates valvular damage due to RHD.

\section{Abbreviations}

RHD

rheumatic heart disease

S1PR1

sphingosine 1-phosphate receptor 1

STAT3

signal transducer and activator of transcription 3

Th17

T helper 17

GAS

Group A streptococci

CFA 
Freund's adjuvant

STAT3-siRNA

STAT3-small interfering RNA

$\mathrm{p}$ -

phosphorylated

ELISA

Enzyme-linked immunosorbent assay

IL

interleukin

RT-qPCR

Reverse transcription-quantitative PCR

WB

Western blotting

RORyt

retinoic acid-related orphan receptor gamma $\mathrm{T}$

NS

normal saline

AAV

adeno-associated viral

$\mathrm{H} \& \mathrm{E}$

haematoxylin and eosin

IHS

immunohistochemical score

DAB

diaminobenzidine

PVDF

polyvinylidene fluoride

ELISA

Enzyme-linked immunosorbent assay

t-

total

COL 1

type 1 collagen

COL3

type 3 collagen

Col3a1

type III a1 chain

FSP1

fibroblast-specific protein 1

MAPKs 
mitogen-activated protein kinases

\section{Declarations}

\section{Ethics approval and consent to participate}

Protocols involving animals were approved by the Medical Ethics Committee of the First Affiliated Hospital of Guangxi Medical University (permit no. 2019-KY-E-034).

\section{Consent for publication}

Not applicable.

\section{Availability of data and materials}

All data generated or analyzed during this study are included in this published article.

\section{Competing interests}

The authors declare that they have no competing interests.

\section{Funding}

The present study was supported by the National Natural Science Foundation of China (grant no. 81660069), Guangxi Key Laboratory Base of Precision Medicine in Cardio-cerebrovascular Disease Control and Prevention (grant no. 17-259-85) and Guangxi Clinical Research Center for Cardiocerebrovascular Diseases (grant no. AD17129014).

\section{Authors' contributions}

Z.Y.Z. and F.H. conceived and designed the study. S.L.X. and A.C. participated in the experimental design. S.L.X., A.C., Y.J.W. and C.H.L. performed the experiments. S.L.X., A.C., H.W. and J.L.W. analyzed the data. S.L.X. wrote the manuscript and all authors read and approved the final manuscript.

\section{Acknowledgements}

Not applicable.

\section{References}

1. DA W, CO J, SM C, et al: Global, Regional, and National Burden of Rheumatic Heart Disease, 19902015. 377: 713-722, 2017.

2. Leal MTBC, Passos LSA, Guarçoni FV, et al. Rheumatic heart disease in the modern era: recent developments and current challenges. Rev Soc Bras Med Trop. 2019;52:e20180041-1. 
3. Mirabel M, Narayanan K, Jouven X, Marijon E. Cardiology patient page. Prevention of acute rheumatic fever and rheumatic heart disease. Circulation. 2014;130:e35-7.

4. Zhao Z, He D, Ling F, et al. CD4(+) T cells and TGF 31 /MAPK signal pathway involved in the valvular hyperblastosis and fibrosis in patients with rheumatic heart disease. Exp Mol Pathol. 2020;114:104402-2.

5. Messias-Reason IJ, Schafranski MD, Kremsner PG, Kun JF. Ficolin 2 (FCN2) functional polymorphisms and the risk of rheumatic fever and rheumatic heart disease. Clin Exp Immunol. 2009;157:395-9.

6. Li M, Xu S, Geng Y, et al. The protective effects of L-carnitine on myocardial ischaemia-reperfusion injury in patients with rheumatic valvular heart disease undergoing CPB surgery are associated with the suppression of NF-kappaB pathway and the activation of Nrf2 pathway. Clin Exp Pharmacol Physiol. 2019;46:1001-12.

7. Hu YS, Han X, Liu XH. STAT3: A Potential Drug Target for Tumor and Inflammation. Curr Top Med Chem. 2019;19:1305-17.

8. Liu X, Hu H, Fan H, et al. The role of STAT3 and AhR in the differentiation of CD4 + T cells into Th17 and Treg cells. Med (Baltim). 2017;96:e6615.

9. Gaffen SL, Jain R, Garg AV, Cua DJ. The IL-23-IL-17 immune axis: from mechanisms to therapeutic testing. Nat Rev Immunol. 2014;14:585-600.

10. Whibley N, Tritto E, Traggiai E, et al. Antibody blockade of IL-17 family cytokines in immunity to acute murine oral mucosal candidiasis. J Leukoc Biol. 2016;99:1153-64.

11. Zhang Y, Shao Z, Zhang X, et al. TIPE2 Play a Negative Role in TLR4-Mediated Autoimmune T Helper 17 Cell Responses in Patients with Myasthenia Gravis. J Neuroimmune Pharmacol. 2015;10:635-44.

12. Bas HD, Baser $K$, Yavuz E, et al. A shift in the balance of regulatory $T$ and $T$ helper 17 cells in rheumatic heart disease. J Investig Med. 2014;62:78-83.

13. Z YW, C Z G, L L and Pathology. LWJCptojotSfC: Changes in the expression of Th17 cell-associated cytokines in the development of rheumatic heart disease. 24: 382-387, 2015.

14. Aoki M, Aoki H, Ramanathan R, Hait NC, Takabe K. Sphingosine-1-Phosphate Signaling in Immune Cells and Inflammation: Roles and Therapeutic Potential. Mediators Inflamm. 2016;2016:86068788.

15. Garris CS, Wu L, Acharya S, et al. Defective sphingosine 1-phosphate receptor 1 (S1P1) phosphorylation exacerbates TH17-mediated autoimmune neuroinflammation. Nature immunology. 2013;14:1166-72.

16. Wu XD, Zeng ZY, Gong DP, Wen JL, Huang F. Potential involvement of S1PR1/STAT3 signaling pathway in cardiac valve damage due to rheumatic heart disease. Biotech Histochem. 2019;94:398403.

17. Chen $A$, Wen J, Lu C, et al. Inhibition of miR-155-5p attenuates the valvular damage induced by rheumatic heart disease. Int J Mol Med. 2020;45:429-40. 
18. Guendisch U, Weiss J, Ecoeur F, et al. Pharmacological inhibition of RORyt suppresses the Th17 pathway and alleviates arthritis in vivo. PLoS One. 2017;12:e0188391-1.

19. Friedrichs K, Gluba S, Eidtmann H, Jonat W. Overexpression of p53 and prognosis in breast cancer. Cancer. 1993;72:3641-7.

20. Wang X-T, Wu X-D, Lu Y-X, et al. Egr-1 is involved in coronary microembolization-induced myocardial injury via Bim/Beclin-1 pathway-mediated autophagy inhibition and apoptosis activation. Aging. 2018;10:3136-47.

21. Purushothaman KR, Purushothaman M, Turnbull IC, et al. Association of altered collagen content and lysyl oxidase expression in degenerative mitral valve disease. Cardiovasc Pathol. 2017;29:11-8.

22. Mortality GBD, Causes of Death C. Global, regional, and national life expectancy, all-cause mortality, and cause-specific mortality for 249 causes of death, 1980-2015: a systematic analysis for the Global Burden of Disease Study 2015. Lancet. 2016;388:1459-544.

23. Collaborators GBDCoD. Global, regional, and national age-sex specific mortality for 264 causes of death, 1980-2016: a systematic analysis for the Global Burden of Disease Study 2016. Lancet. 2017;390:1151-210.

24. Collaborators GBDCoD. Global, regional, and national age-sex-specific mortality for 282 causes of death in 195 countries and territories, 1980-2017: a systematic analysis for the Global Burden of Disease Study 2017. Lancet. 2018;392:1736-88.

25. Camporeale A, Marino F, Papageorgiou A, et al. STAT3 activity is necessary and sufficient for the development of immune-mediated myocarditis in mice and promotes progression to dilated cardiomyopathy. EMBO Mol Med. 2013;5:572-90.

26. Yuan J, Yu M, Lin Q-W, et al. Th17 cells contribute to viral replication in coxsackievirus B3-induced acute viral myocarditis. J Immunol. 2010;185:4004-10.

27. Kurdi M, Zgheib C, Booz GW. Recent Developments on the Crosstalk Between STAT3 and Inflammation in Heart Function and Disease. Front Immunol. 2018;9:3029-9.

28. Turkson J, Jove R. STAT proteins: novel molecular targets for cancer drug discovery. Oncogene. 2000;19:6613-26.

29. Zhang D, Liu X, Chen X, et al. Role of the MAPKs/TGF-beta1/TRAF6 signaling pathway in atrial fibrosis of patients with chronic atrial fibrillation and rheumatic mitral valve disease. Cardiology. 2014;129:216-23.

30. Lu D, Liu L, Ji X, et al. The phosphatase DUSP2 controls the activity of the transcription activator STAT3 and regulates TH17 differentiation. Nature immunology. 2015;16:1263-73.

31. Bilik MZ, Kaplan I, Polat N, et al. Serum Levels of IL-17 and IL-23 in Patients With Rheumatic Mitral Stenosis. Medicine. 2016;95:e3562-2.

32. Huang N, Liu K, Liu J, et al. Interleukin-37 alleviates airway inflammation and remodeling in asthma via inhibiting the activation of NF-KB and STAT3 signalings. Int Immunopharmacol. 2018;55:198204. 
33. Gao W, McCormick J, Connolly M, Balogh E, Veale DJ, Fearon U. Hypoxia and STAT3 signalling interactions regulate pro-inflammatory pathways in rheumatoid arthritis. Ann Rheum Dis. 2015;74:1275-83.

34. Liu J, Fei D, Xing J, Du J. MicroRNA-29a inhibits proliferation and induces apoptosis in rheumatoid arthritis fibroblast-like synoviocytes by repressing STAT3. Biomed Pharmacother. 2017;96:173-81.

35. Lee SH, Kim E-K, Kwon J-E, et al. Ssu72 attenuates autoimmune arthritis via targeting of STAT3 signaling and Th17 activation. Sci Rep. 2017;7:5506-6.

36. Yu H, Pardoll D, Jove R. STATs in cancer inflammation and immunity: a leading role for STAT3. Nat Rev Cancer. 2009;9:798-809.

37. Remenyi B, ElGuindy A, Smith SC Jr, Yacoub M, Holmes DR, Jr.: Valvular aspects of rheumatic heart disease. Lancet 387: 1335-1346, 2016.

\section{Supplementary Figures}

1. S1PR1 original gel. The marker is on the far right, and developed. Cropping is to remove the marker.

2. Tubulin for S1PR1 normalization. This picture is not in the final figure. This is for S1PR1's normalizing.

3. STAT3 original gel. The marker is on the far right, developed. The cropped part is 4-7 squares from right to left.

4. P-STAT3 original gel. The marker is on the far right, undeveloped. The cropped part is 4-7 squares from right to left.

5. Tubulin original gel. The marker is on the far right, not included. The cropped part is 4-7 squares from right to left.

6. STAT3 early experiment. The marker is on the far right, developed. This picture is only attached to reflect the development of the marker, this is not the picture used in the final figure.

\section{Figures}


A

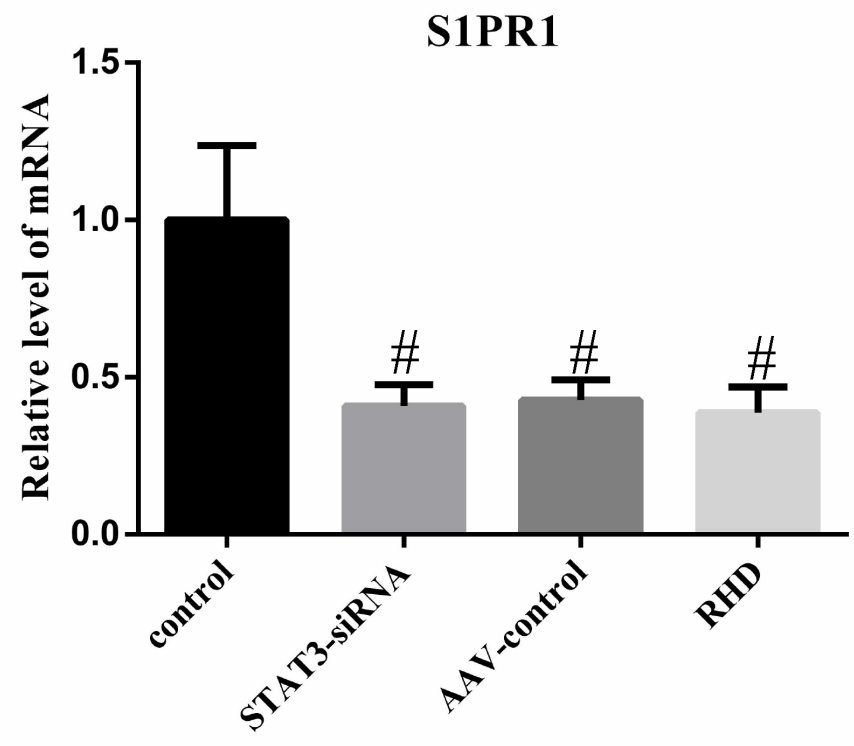

B

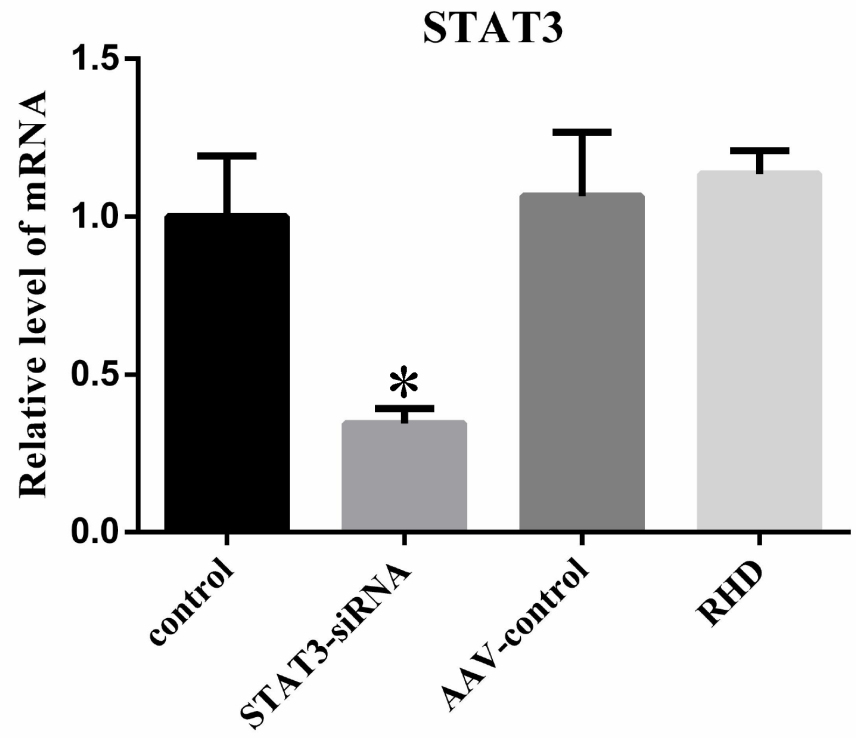

Figure 1

Reverse transcription-quantitative PCR. (A) Fold change of S1PR1 mRNA expression among 4 groups. (B) Fold change of STAT3 mRNA expression among 4 groups. This figure showed that the mRNA expression of S1PR1 in control group was higher that other three groups, and the mRNA expression of STAT3 in STAT3-siRNA group was lower than that in RHD group. Data was shown as the mean \pm standard deviation; $\# \mathrm{P}<0.05$ compared to the control group. ${ }^{*} \mathrm{P}<0.05$ compared to the RHD group. 
A

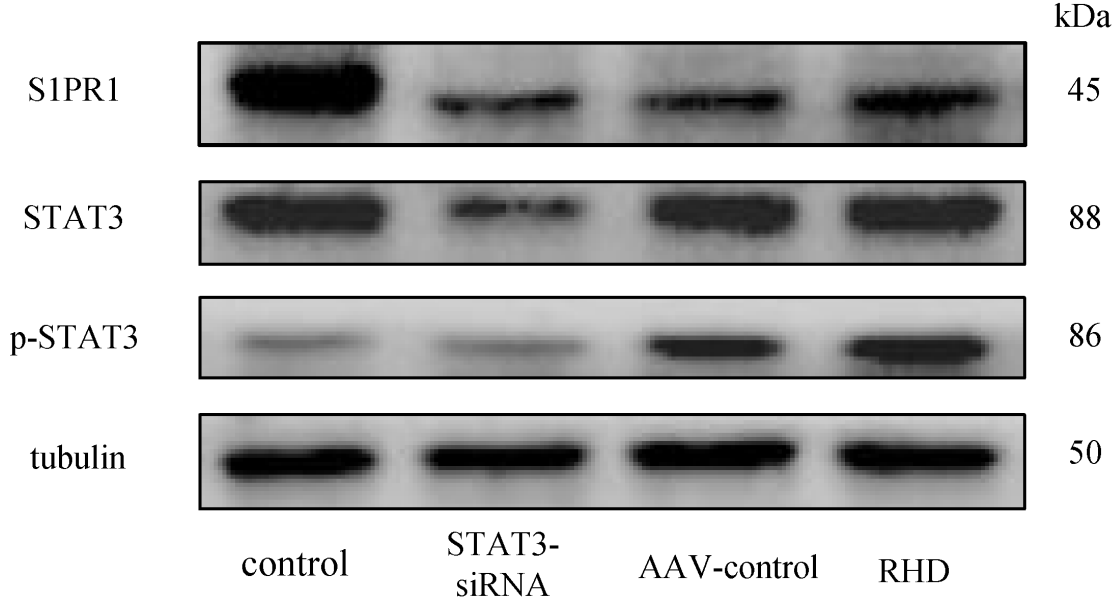

C

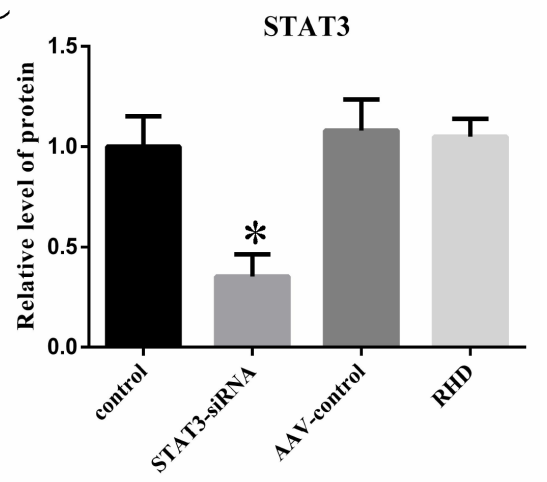

B
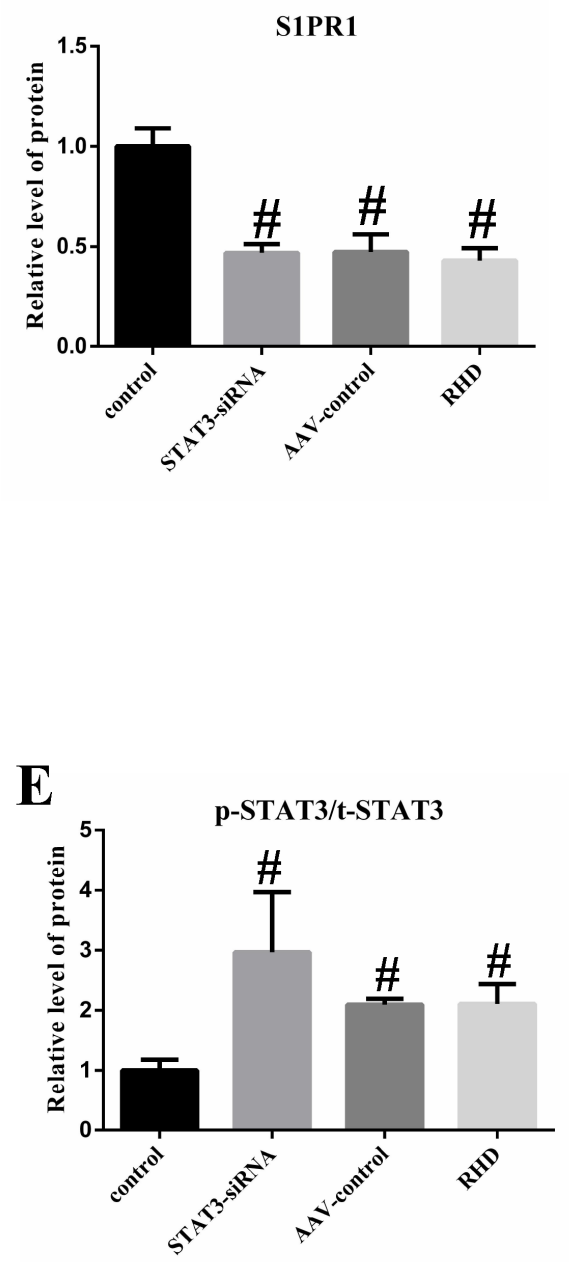

Figure 2

Western blot analysis. (A) The protein bands of S1PR1, STAT3 and p-STAT3 in the four groups. (B) Fold change of S1PR1 protein expression among 4 groups. (C) Fold change of STAT3 protein expression among 4 groups. (D) Fold change of p-STAT3 protein expression among 4 groups. (E) The relative protein level of the ratio of p-STAT3/t-STAT3. This figure showed that the protein expression of S1PR1 in control group was higher that other three groups, and the inhibition of STAT3 by STAT3-siRNA decreased the protein expression of STAT3 and the total amount of p-STAT3 in the valve tissue. Data was shown as the mean \pm standard deviation; $\# \mathrm{P}<0.05$ compared to the control group. ${ }^{*}<<0.05$ compared to the RHD group. 

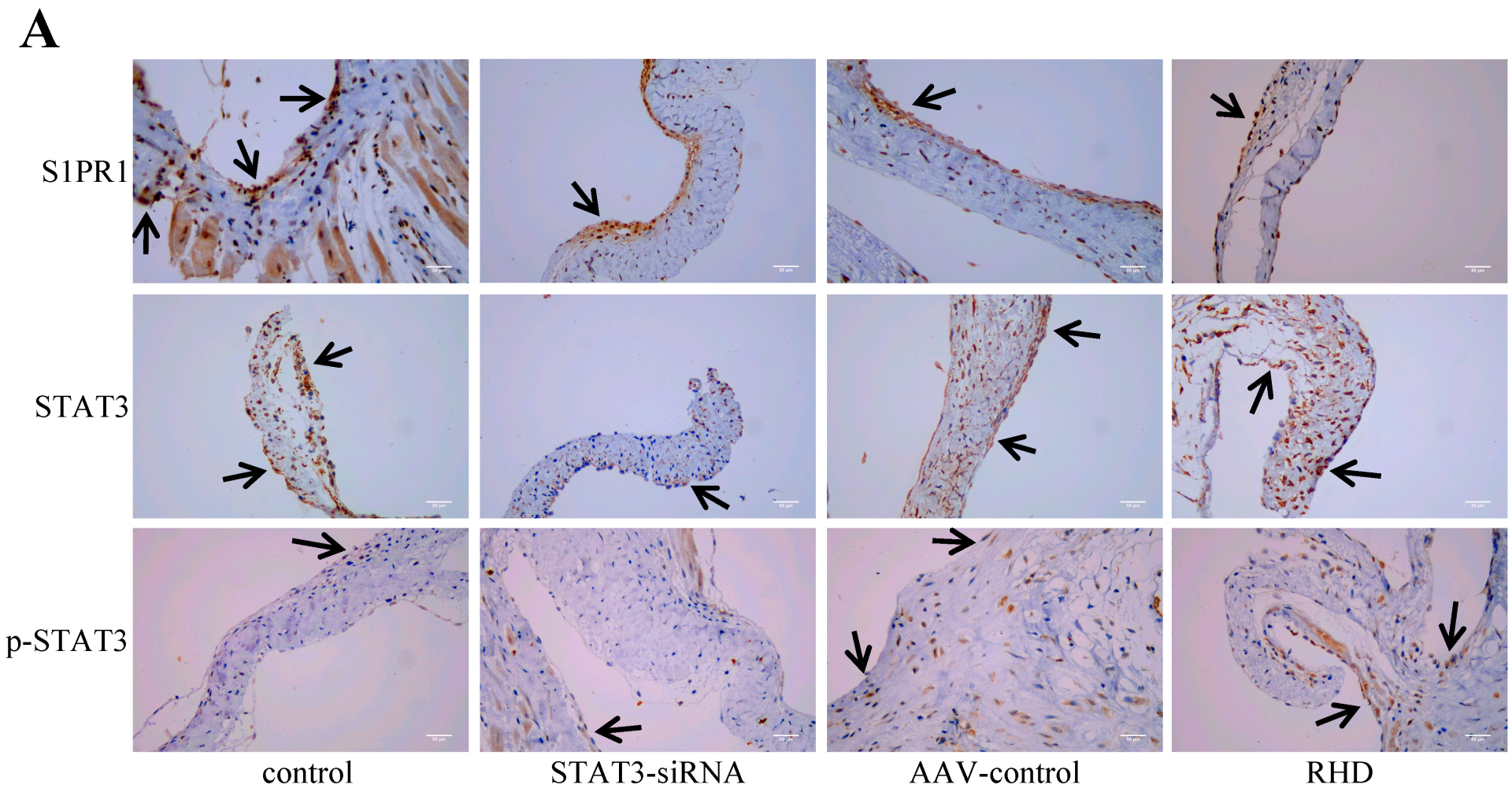

STAT3-siRNA

AAV-control

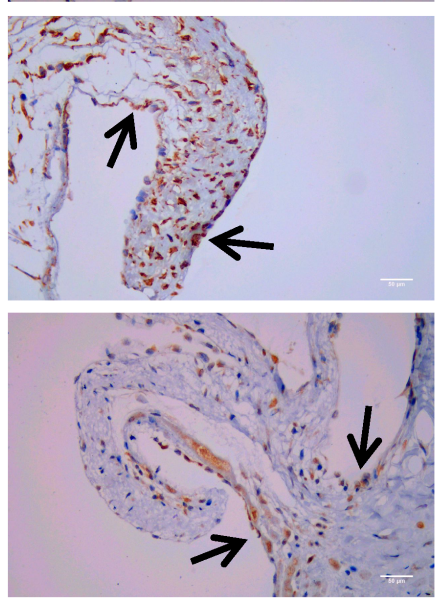

RHD
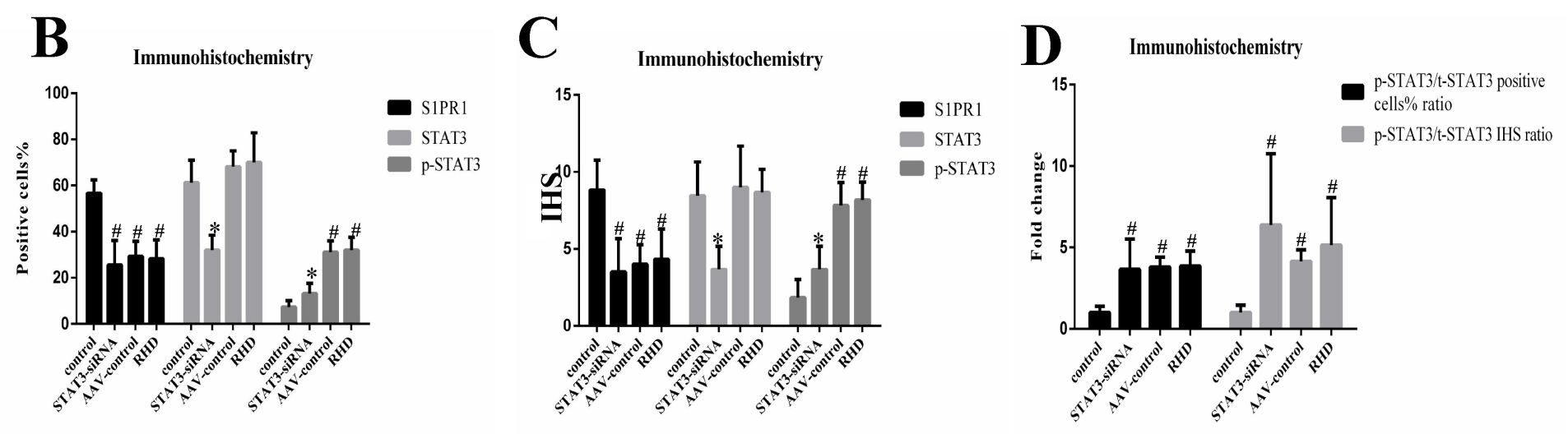

Figure 3

Immunohistochemistry analysis results of S1PR1, STAT3 and p-STAT3. (A) Immunohistochemical analysis results of S1PR1, STAT3 and p-STAT3 in valve tissues; magnification, $x 400$; scale bar: $50 \mu \mathrm{m}$; the arrows marked the positive staining results. (B) The positive cells percentage. (C) The IHS. (D) The ratio of p-STAT3/t-STAT3. This figure showed that the protein expression of S1PR1 in control group was higher that other three groups, and the inhibition of STAT3 by STAT3-siRNA decreased the protein expression of STAT3 and the total amount of p-STAT3 in the valve tissue. Data was shown as the mean \pm standard deviation; $\# \mathrm{P}<0.05$ compared to the control group. ${ }^{*} \mathrm{P}<0.05$ compared to the $\mathrm{RHD}$ group. 
A
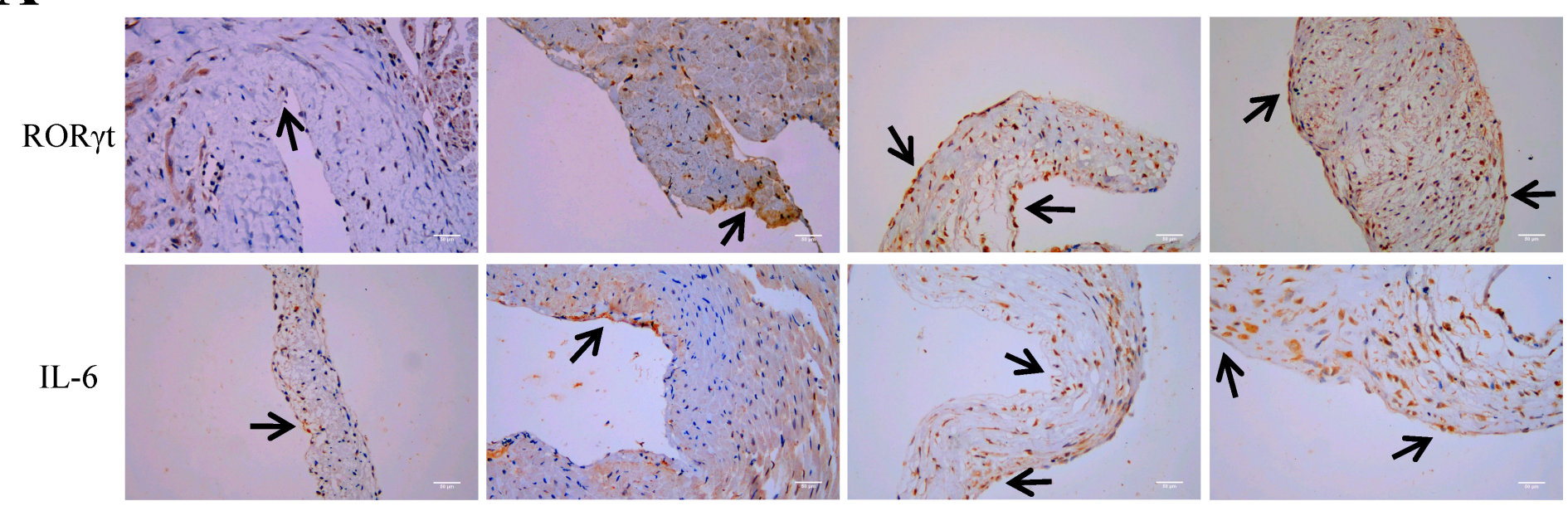

IL-17
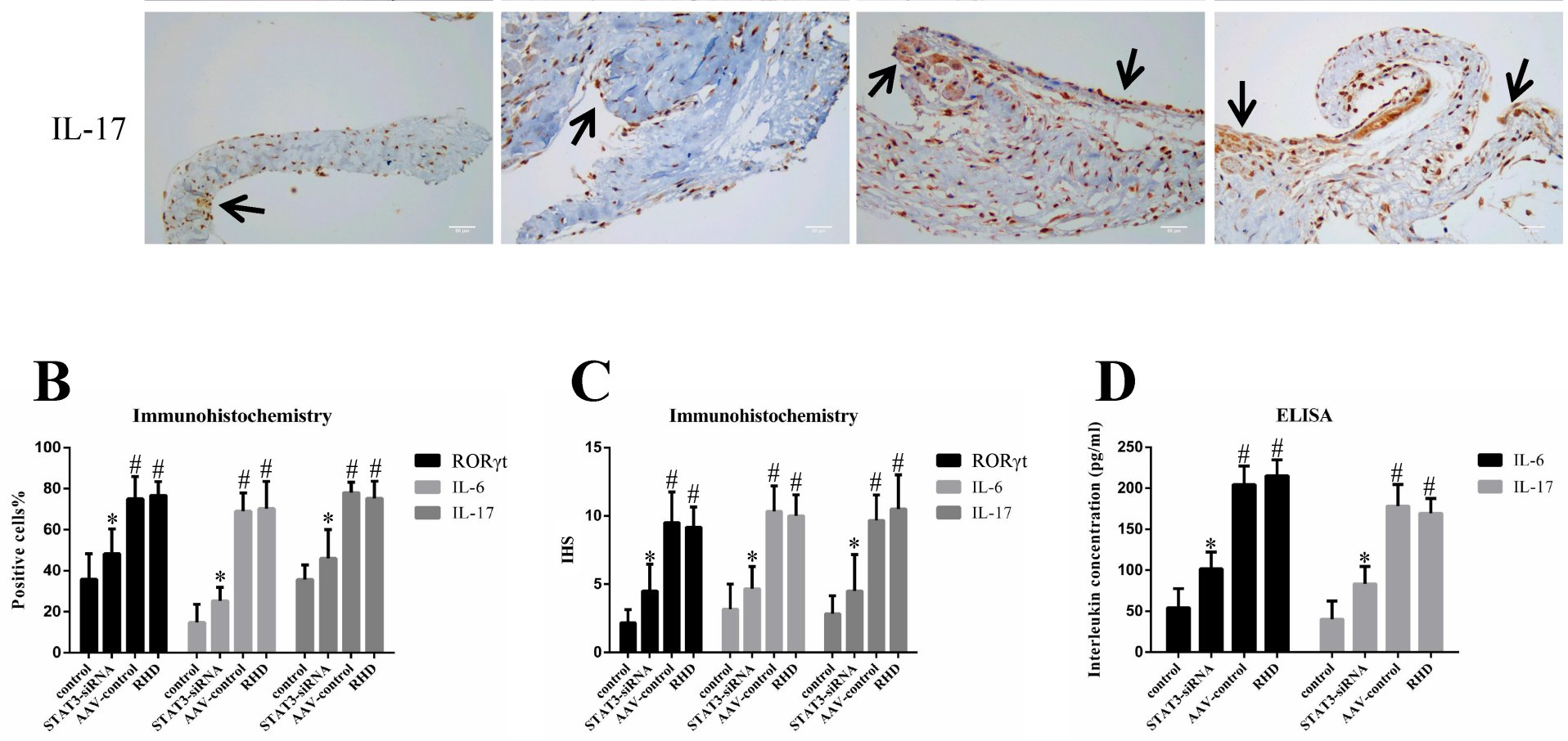

\section{Figure 4}

Th17 cell-related transcription factor and cytokines. (A) Immunohistochemical analysis results of RORyt, IL-6 and IL-17 in valve tissues; magnification, $x 400$; scale bar: $50 \mu \mathrm{m}$; the arrows marked the positive staining results. (B) The positive cells percentage. (C) The IHS. (D) ELISA analysis for IL-6 and IL-17 in serum. This figure showed that the expression of RORYt, IL-6 and IL-17 was attenuated in STAT3-siRNA group. Data was shown as the mean \pm standard deviation; $\# \mathrm{P}<0.05$ compared to the control group. ${ }^{*} \mathrm{P}<0.05$ compared to the RHD group. 
A

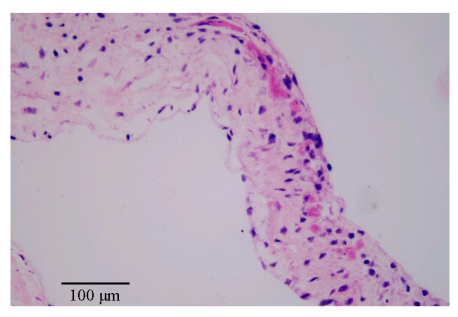

control

B

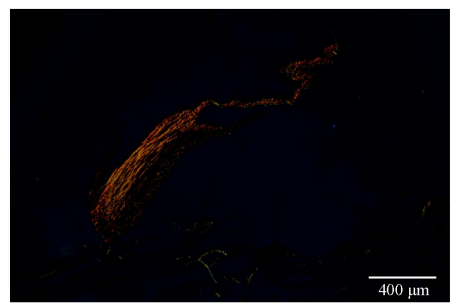

control

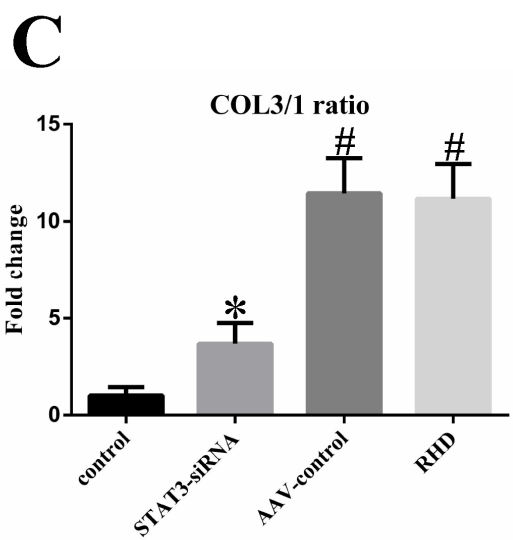

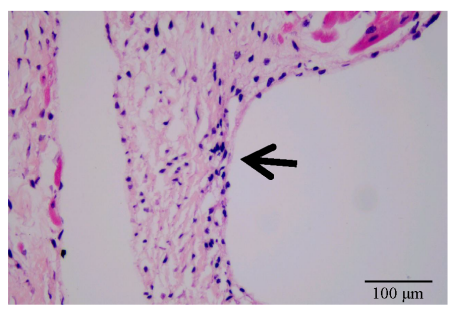

STAT3-siRNA

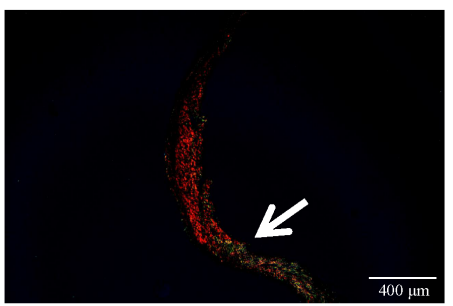

STAT3-siRNA

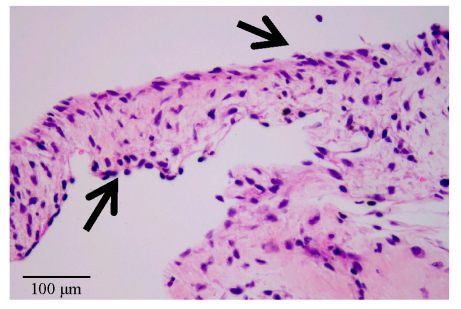

AAV-control

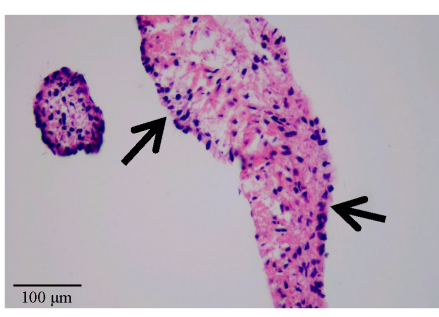

RHD

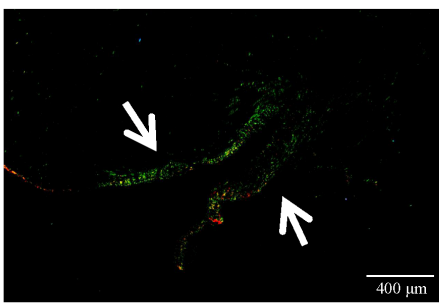

AAV-control

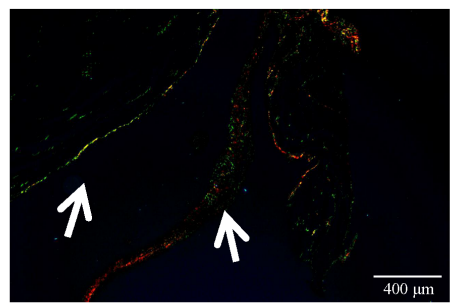

RHD
D

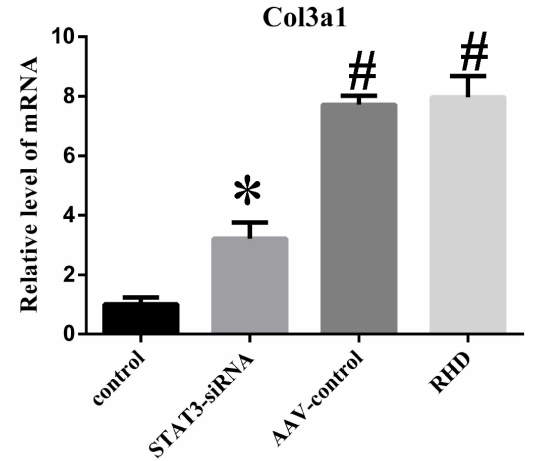

E

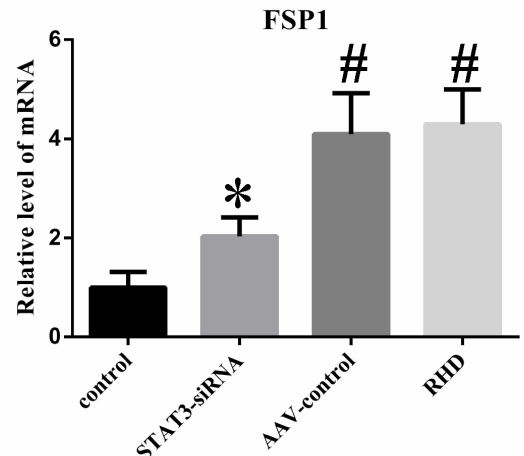

Figure 5

$H \& E$, Sirius red staining of valve tissues and RT-qPCR analysis of fibrosis-related factors. (A) H\&E staining; there was an inflammatory response in the heart valves of the rats in the AAV-control and RHD groups. In the STAT3-siRNA group, inhibition of STAT3 by STAT3-siRNA reduced inflammatory response; magnification, x400; scale bar: $100 \mu \mathrm{m}$; the arrows marked inflammatory response. (B) Sirius red staining, fibrosis was observed in AAV-control and RHD groups. Inhibition of STAT3 by STAT3-siRNA reduced the degree of fibrosis; magnification, x100; scale bar: $400 \mu \mathrm{m}$; the arrows marked COL3. (C) COL3/1 ratio was significantly higher in RHD group than in control group, inhibition of STAT3 by STAT3-siRNA reduced the COL3/1 ratio. (D) \& (E) RT-qPCR analysis of Col3a1 and FSP1; both Col3a1 mRNA expression and FSP1 mRNA expression were significantly higher in RHD group than in control group, and inhibition of STAT3 by STAT3-siRNA could reduce all of them. Data was shown as the mean \pm standard deviation; \#P<0.05 compared to the control group. ${ }^{*} \mathrm{P}<0.05$ compared to the RHD group. 


\section{Supplementary Files}

This is a list of supplementary files associated with this preprint. Click to download.

- ARRIVEchecklist.docx

- pSTAT3originalgel.tif

- S1PR1originalgel.tif

- STAT3earlyexperiment.tif

- STAT3originalgel.tif

- tubulinforS1PR1normalization.tif

- tubulinoriginalgel.tif 